МАТЕМАТИЧЕСКОЕ МОДЕЛИРОВАНИЕ И ИНФОРМАТИКА

DOI 10.15507/2079-6900.21.201902.259-268

MSC201093C95

\title{
Empirical and physics-based approaches to estimate states of lithium-ion battery
}

(c) A. A. Fedorova ${ }^{1}$

\begin{abstract}
Lithium-ion batteries are integral parts of our life due to the rapid increase of applications which require batteries for their exploitation. Thus, there is a market demand to produce lithium-ion batteries for a huge number of applications from electric vehicles to energy storages. Battery Management System (BMS) is developed to maintain safe battery exploitation conditions. Most BMSs are embedded systems that have physical memory limits. Therefore, battery model should be easy to simulate to be integrated into BMS for states estimation. In the present paper we intend to compare empirical and physics-based approaches to estimate lithium-ion battery states with respect to their possibility of implementation in the embedded system. We will use Kalman filter to estimate battery states by means of the mentioned models.

Key words: lithium-ion battery, equivalent-circuit model, porous-electrode model, reduced-order model, Kalman filter
\end{abstract}

\section{Introduction}

The number of lithium-ion batteries applications increases annually. Thus, there is a great demand for development of batteries monitoring algorithms to diagnose and prevent undesirable battery damage and predict their state.

There are many battery states to consider. They include State-of-Charge (SOC), State-of-Health (SOH), State-of-Power (SOP), State-of-Function (SOF), etc. Lithium-ion battery SOC is the percentage of the maximum possible charge that is present inside a rechargeable battery [1]. SOH represents battery's ability to store and deliver electrical energy, compared with a new battery [2]. SOP is a quantity that describes the battery's power capability [3]. SOF is a figure of merit that describes the battery capability to perform a certain task [4].

As for today there is no equipment to measure any of the states directly from the lithium-ion battery cell. However, we can measure current, voltage and temperature from any cell. Many articles describe different techniques to estimate states of lithium-ion battery based on observed data.

Nejad et al. estimated SOC of lithium-ion iron phosphate cell with flat open-circuit voltage (OCV) curve by means of equivalent-circuit model (ECM) and two separate Extended Kalman Filters [5]. The main advantage of their work is ability to estimate SOC in a real time mode despite flatness of the OCV curve.

Various papers consider estimation and prediction of lithium-ion battery SOH. The U-D factorization-based RLS method and ECM were used in [6] to estimate capacity of lithium-ion batteries in PHEV/EV applications. Richardson et al. [7] presented approach to predict shortterm and long-term battery degradation behaviour by means of Gaussian process regression.

\footnotetext{
${ }^{1}$ Anna A. Fedorova, Postgraduate Student, Department of Control Theory, Faculty of Applied Mathematics-Control Processes, St. Petersburg State University (35 Universitetskaya Emb, Petergof, SaintPetersburg, Russia, 198504), ORCID: http://orcid.org/0000-0002-0864-7947, anyfyodorova@gmail.com
} 
The main bottleneck of their approach is the computational cost of handling large numbers of outputs. However, presented method has reasonable accuracy to predict future capacity 10 cycles ahead based on the requirements of the system to facilitate corrective action.

Particle Swarm Optimization and Unscented Kalman Filter were implemented in [8] to estimate lithium-ion battery SOP. The authors showed the ability of their approach to work not only for the laboratory-based applications but for real time ones as well.

Practically all of the above-mentioned references employ ECM or empirical-based approach to estimate states of lithium-ion battery. However, at the present time many attempts are made to implement electrochemical models of lithium-ion batteries in the embedded BMS. Particularly, Smith et al. [9] made one of the first attempts to convert full-order model of ideal lithium-ion battery cell [10] to the reduced-order state-space representation that can be easily implemented in the BMS.

In the following paper we intend to compare results of empirical and physics-based approaches for battery states estimation. We will present all the necessary systems of ordinary and partially differential equations to simulate battery behaviour in sections 2 and 3 . In section 4 we will show how to estimate lithium-ion battery SOC and SOH based on the aforementioned models. In section 5 of the paper we will compare estimation results by means of the dataset that includes current and voltage measurements.

\section{Empirical approach}

Empirical modelling or ECM allows simulating lithium-ion battery behaviour using common circuit elements (capacitor, resistor, etc.) These models enable fast and robust simulations without high physical memory requirements. That is why they are implemented in most BMS now. ECM provides accurate results regarding input-output (current-voltage) matching of the battery. Moreover, ECM is actively used now for estimation and prediction of lithium-ion battery $\mathrm{SOH}[11]-[14]$.

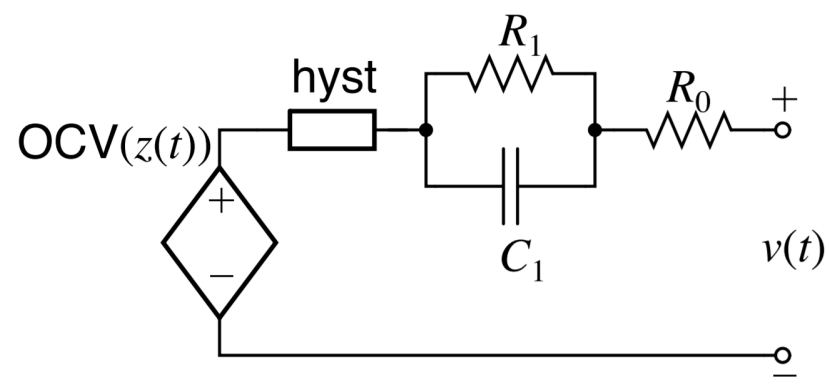

F i g. $\quad 2.1$

Example of Equivalent-Circuit Model

Let us consider one of the common ECM that is represented in the figure above [15]. The state-space system of such model is the following set of equations

$$
\begin{aligned}
{\left[\begin{array}{c}
z_{k+1} \\
i_{R_{1}, k+1} \\
h_{k+1}
\end{array}\right] } & =\left[\begin{array}{ccc}
1 & 0 & 0 \\
0 & A_{R C} & 0 \\
0 & 0 & A_{H_{k}}
\end{array}\right]\left[\begin{array}{c}
z_{k} \\
i_{R_{1}, k} \\
h_{k}
\end{array}\right]+\left[\begin{array}{cc}
-\frac{\eta_{k} \Delta t}{Q} & 0 \\
B_{R C} & 0 \\
0 & \left(1-A_{H_{k}}\right)
\end{array}\right]\left[\begin{array}{c}
i_{k} \\
\operatorname{sgn}\left(i_{k}\right)
\end{array}\right], \\
v_{k} & =O C V\left(z_{k}\right)+M h_{k}-\sum_{i} R_{i} i_{R_{i}, k}-R_{0} i_{k}
\end{aligned}
$$


where $z_{k}$ is the battery SOC at discrete-time index $k, \eta_{k}$ is the unitless cell coulombic efficiency at time $k, i_{k}$ is the input current at time $k[\mathrm{~A}], \Delta t$ is the sample period [s], $Q$ is the cell total capacity [Ah]. Current through the resistor $R_{1}$ in the resistor-capacitor network at discrete-time index $k$ is denoted as $i_{R_{1}, k}$. This term models the slow time constants of diffusion processes occurring within the cell. Note that $A_{R C}=\exp \left(\frac{-\Delta t}{R_{1} C_{1}}\right)$ and $B_{R C}=1-\exp \left(\frac{-\Delta t}{R_{1} C_{1}}\right) . h_{k}$ is hysteresis at discrete-time index $k \cdot A_{H_{k}}=\exp \left(-\left|\frac{\eta_{k} i_{k} \gamma \Delta t}{Q}\right|\right)$, where $\gamma$ is the unitless constant that adjusts how quickly does the hysteresis state change with a change in cell SOC. $v_{k}$ is battery terminal voltage [V]. $O C V$ is the battery open-circuit voltage [V], $M$ is the maximum absolute analogue hysteresis voltage $[\mathrm{V}], M_{0}$ is the instantaneous hysteresis voltage $[\mathrm{V}], R_{0}$ is the pure ohmic resistance of the battery $[\Omega]$.

Despite the ECM advantages mentioned at the beginning of the current section, empirical modelling does not provide any information regarding internal electrochemical state of lithiumion battery. Moreover, ECM does not allow to extrapolate battery state. All the referred facts were the beginning of the development of the reduced-order physics-based models of lithium-ion batteries. Let us consider them in detail in the following section.

\section{Physics-based approach}

Physics-based models are developed based on the main electrochemical reactions that take place inside lithium-ion battery cell during charge, discharge and rest. In this section we will consider the set of partial differential equations (PDEs) that allows describing electrochemical cell behaviour and was derived firstly by Newman et al [10], [16]. Furthermore, we will present an approach to convert the set of PDEs to the set of ordinary differential equations (ODEs) that is similar in computational complexity to the ECM considered in the previous section [17].

\subsection{Porous electrode model of lithium-ion cell}

The first electrochemical approach to porous electrodes modelling for battery applications was presented in 1975. In the porous electrode theory, the electrode is treated as a superposition of two continua, namely, the electrolytic solution and the solid matrix. The solid matrix is modelled as microscopic spherical particles where the lithium ions diffuse and react on the spheres surface. This approach was later expanded to two composite electrodes and a separator in 1994 [18].

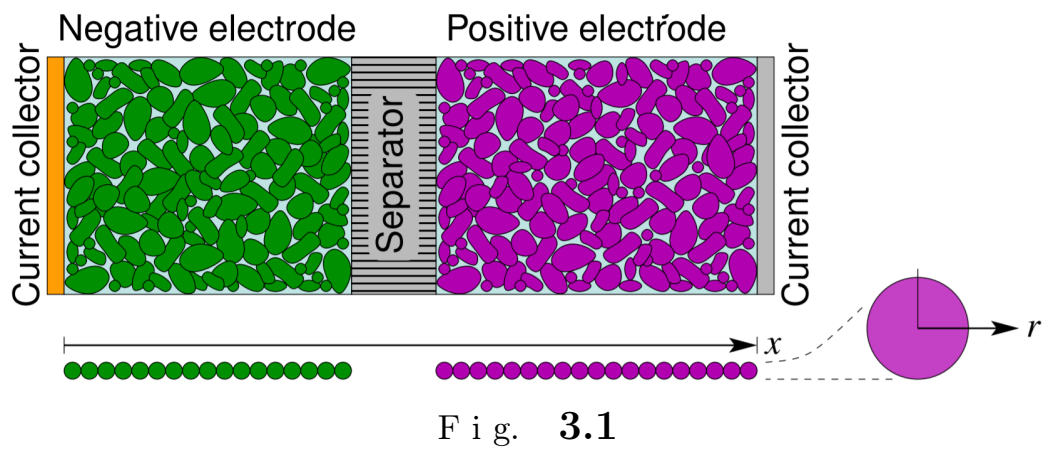

Pseudo-two-dimensional porous-electrode model [19]

We will present here all the necessary PDEs and boundary conditions to model lithiumion cell at the porous electrode scale according to [10]. Figure above depicts the geometry of a lithium-ion cell cross section; the model $x$ dimension spans the cell width, while the 
model $r$ pseudo-dimension is measured outward from a particle center and describes lithium concentration inside solid particles (resulting in a «pseudo-two dimensional» model) [19]. The porous-electrode model includes the electrode-scale variables: reaction flux $j$, potential in the solid phase $\phi_{s}$, concentration of lithium in the solid phase $c_{s}$; and the cell-scale variables: potential in the electrolyte phase $\phi_{e}$, concentration of lithium in the electrolyte phase $c_{e}$.

1. The solid-phase mass conservation

$$
\frac{\partial c_{s}(r, x, t)}{\partial t}=\frac{D_{s}}{r^{2}} \frac{\partial}{\partial r}\left(r^{2} \frac{\partial c_{s}(r, x, t)}{\partial r}\right)
$$

the boundary conditions to this PDE are

$$
D_{s} \frac{\partial c_{s}(0, x, t)}{\partial r}=0, \quad D_{s} \frac{\partial c_{s}\left(R_{s}, x, t\right)}{\partial r}=-j(x, t),
$$

where $j(x, t)$ is a measure of the amount of lithium moving across the boundary of the solid particle. The initial condition is

$$
c_{s}(r, x, 0)=c_{s, 0}, \quad 0 \leq r \leq R_{s} .
$$

2. Mass conservation in the electrolyte phase of the porous electrode

$$
\frac{\partial\left(\varepsilon_{e} c_{e}(x, t)\right)}{\partial t}=\frac{\partial}{\partial x}\left(D_{\mathrm{e}, \mathrm{eff}} \frac{\partial}{\partial x} c_{e}(x, t)\right)+a_{s}\left(1-t_{+}^{0}\right) j(x, t),
$$

where the boundary conditions are given by

$$
\frac{\partial c_{e}(0, t)}{\partial x}=\frac{\partial c_{e}(L, t)}{\partial x}=0
$$

and the initial concentration of lithium in the electrolyte is

$$
c_{e}(x, 0)=c_{e, 0}, \quad 0 \leq x \leq L .
$$

3. Charge conservation in the solid phase of the porous electrode

$$
\frac{\partial}{\partial x}\left(\sigma_{\mathrm{eff}} \frac{\partial}{\partial x} \phi_{s}(x, t)\right)=a_{s} F j(x, t),
$$

the boundary conditions are

$$
-\sigma_{\mathrm{eff}} \frac{\partial \phi_{s}(0, t)}{\partial x}=\sigma_{\mathrm{eff}} \frac{\partial \phi_{s}(L, t)}{\partial x}=\frac{I}{A}=i_{\mathrm{app}} .
$$

4. Charge conservation in the electrolyte phase of the porous electrode

$$
\frac{\partial}{\partial x}\left(\kappa_{\mathrm{eff}} \frac{\partial}{\partial x} \phi_{e}(x, t)+\kappa_{D, \mathrm{eff}} \frac{\partial}{\partial x} \ln c_{e}(x, t)\right)=-a_{s} F j(x, t),
$$

where

$$
\kappa_{\text {eff }}=\kappa \varepsilon_{e, \text { brug }} \quad \text { and } \quad \kappa_{D, \text { eff }}=\frac{-2 R T}{F} \kappa_{\text {eff }}\left(1-t_{+}^{0}\right)\left(1+\frac{\operatorname{d} \ln f_{ \pm}}{\operatorname{dln} c_{e}}\right)
$$

with the boundary conditions

$$
\frac{\partial \phi_{e}(0, t)}{\partial x}=\frac{\partial \phi_{e}(L, t)}{\partial x}=0
$$


5. Lithium movement between solid and electrolyte phases

$$
j(x, t)=k c_{e}^{1-\alpha}\left(c_{s, \max }-c_{s, e}\right)^{1-\alpha} c_{s, e}^{\alpha}\left\{\exp \left(\frac{(1-\alpha) F}{R T} \eta\right)-\exp \left(\frac{-\alpha F}{R T} \eta\right)\right\},
$$

where the overpotential $\eta$ is

$$
\eta=\phi_{s}-\phi_{e}-U_{\mathrm{ocp}}-j F R_{\mathrm{film}} .
$$

All variables used in the above equations are described in the nomenclature at the end of the present article.

\subsection{Reduced-order model of lithium-ion cell}

To convert full-order porous electrode model of lithium-ion cell we follow the approach presented in [17] and [20]. We will present the main results of the mentioned articles in the present paper.

The authors developed a one-dimensional physics-based reduced-order model for lithiumion cell. They obtained the set of electrochemical-variable transfer functions to model cell parameters at any desired sets of spatial locations in the cell based only on the cell input current $i_{\text {app }}$ for the following variables: solid potential $\phi_{s}$, electrolyte potential $\phi_{e}$, electrolyte concentration $c_{e}$, solid surface concentration $c_{s}$, reaction flux $j$ and overpotential $\eta$.

The discrete-time realization algorithm [20] is used to produce an optimal reduced-order discrete-time state-space model from these transfer functions, overcoming the limitations of non-linear optimization approaches.

\section{Kalman filter}

Kalman filter is a recursive algorithm used to estimate the state vector of a linear system. If the system is non-linear, it can be linearised at any given time, using the assumption of the explicit dependence of the output characteristics of the system on time. The linear systems for lithium-ion cell were presented in the sections 2 and 3. In this section we examine general steps of the Kalman filter to estimate states of the battery [19], [15].

Let us consider discrete-time linear model of the lithium-ion cell in the following form:

$$
\begin{aligned}
x_{k+1} & =f_{k}\left(x_{k}, u_{k}, w_{k}\right), \\
y_{k} & =h_{k}\left(x_{k}, u_{k}, v_{k}\right),
\end{aligned}
$$

where $u_{k}$ is known input signal (current), $x_{k}$ is the model state vector (SOC), $y_{k}$ is measurable model output (voltage), $w_{k}$ is process-noise random input, $v_{k}$ is sensor-noise random input.

Below we present the main steps of the Kalman filter as applied to the discrete-time linear model considered above. The following notations are used: the decoration $\widehat{(\cdot)}$ indicates extrapolated values of the variable, the decoration $\widetilde{(\cdot)}$ is used to denote estimation error of the variable, $(\cdot)^{-}$indicates extrapolated values of the variable based only on measured data, $(\cdot)^{+}$ is used to mark estimated values of the variable based only on measured data.

\section{Six steps of the Kalman filter}

1. State prediction time update

$$
\widehat{x}_{k}^{-}=\mathbb{E}\left[f\left(x_{k-1}, u_{k-1}, w_{k-1}\right) \mid \mathbb{Y}_{k-1}\right] \approx f\left(\widehat{x}_{k-1}^{+}, u_{k-1}, \bar{w}_{k-1}\right)
$$

where $\bar{w}_{k-1}=\mathbb{E}\left[w_{k-1}\right]$. 
2. Error covariance time update

$$
\begin{aligned}
\widetilde{x}_{k}^{-} & =x_{k}-\widehat{x}_{k}^{-}=f\left(x_{k-1}, u_{k-1}, w_{k-1}\right)-f\left(\widehat{x}_{k-1}^{+}, u_{k-1}, \bar{w}_{k-1}\right), \\
\Sigma_{\widetilde{x}, k}^{-} & =\mathbb{E}\left[\left(\widetilde{x}_{k}^{-}\right)\left(\widetilde{x}_{k}^{-}\right)^{T}\right] \approx \widehat{A}_{k-1} \Sigma_{\widetilde{x}, k-1}^{+} \widehat{A}_{k-1}+\widehat{B}_{k-1} \Sigma_{\widetilde{w}} \widehat{B}_{k-1}^{T},
\end{aligned}
$$

where $\widehat{A}_{k}=\left.\frac{d f_{k}\left(x_{k}, u_{k}, w_{k}\right)}{d x_{k}}\right|_{x_{k}=\widehat{x}_{k}^{+}}$and $\widehat{B}_{k}=\left.\frac{d f_{k}\left(x_{k}, u_{k}, w_{k}\right)}{d w_{k}}\right|_{w_{k}=\bar{w}_{k}}$.

3. Predict system output

$$
\widehat{y}_{k}=\mathbb{E}\left[h\left(x_{k}, u_{k}, v_{k}\right) \mid \mathbb{Y}_{k-1}\right] \approx h\left(\widehat{x}_{k}^{-}, u_{k}, \bar{v}_{k}\right)
$$

where $\bar{v}_{k}=\mathbb{E}\left[v_{k}\right]$.

4. Estimator Kalman gain matrix $L_{k}$

$$
\begin{aligned}
\widetilde{y}_{k} & =y_{k}-\hat{y}_{k}=h\left(x_{k}, u_{k}, v_{k}\right)-h\left(\hat{x}_{k}^{-}, u_{k}, \bar{v}_{k}\right), \\
L_{k} & =\Sigma_{\widetilde{x}, k}^{-} \widehat{C}_{k}^{T}\left[\widehat{C}_{k} \Sigma_{\widetilde{x}, k}^{-} \widehat{C}_{k}^{T}+\widehat{D}_{k} \Sigma_{\widetilde{v}} \widehat{D}_{k}^{T}\right]^{-1},
\end{aligned}
$$

where $\widehat{C}_{k}=\left.\frac{d h_{k}\left(x_{k}, u_{k}, v_{k}\right)}{d x_{k}}\right|_{x_{k}=\widehat{x}_{k}^{-}}$and $\widehat{D}_{k}=\left.\frac{d h_{k}\left(x_{k}, u_{k}, v_{k}\right)}{d v_{k}}\right|_{v_{k}=\bar{v}_{k}}$.

5. State estimate measurement update

$$
\widehat{x}_{k}^{+}=\widehat{x}_{k}^{-}+L_{k}\left(y_{k}-\widehat{y}_{k}\right) .
$$

6. Error covariance measurement update

$$
\Sigma_{\widetilde{x}, k}^{+}=\Sigma_{\widetilde{x}, k}^{-}-L_{k} \Sigma_{\widetilde{y}, k} L_{k}^{T}
$$

\section{Simulation and results}

We now present results of Kalman filter implementation for two lithium-ion cell models: ECM (Section 2) and physics-based (Section 3). We consider 20.5 Ah NMC lithium-ion cell. We use the same set consisting of current and voltage measurements to compare the results (see figure below). All data are available in open-access [19], [15]. MATLAB was used to implement all necessary calculations.
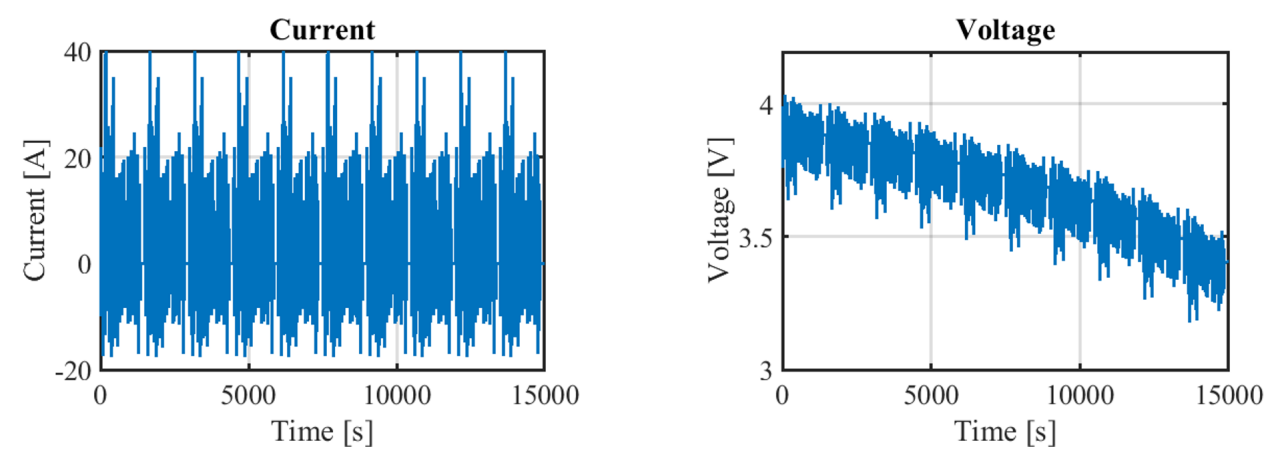

F i g. $\quad 5.1$

A. A. Fedorova. Empirical and physics-based approaches to estimate states of lithium-ion battery 
Current and voltage measurements

As a first step we focus on SOC estimation as the main battery state. SOC is included explicitly into ECM model and can be evaluated from internal electrochemical variables via the following formula:

$$
\mathrm{SOC}_{k}=\frac{\theta_{k}-\theta_{0 \%}}{\theta_{100 \%}-\theta_{0 \%}}
$$

where $\theta_{k}=\frac{c_{s, \text { avg }}}{c_{s, \max }}$ is the present lithium concentration stoichiometry, $\theta_{100 \%}$ and $\theta_{0 \%}$ are its maximum and minimum limits, respectively.

Thus, we have Kalman filter (Section 4) and two linear systems that allow estimating SOC of lithium-ion cell. As it was mentioned at the beginning of the present paper, we concerned about physical memory and speed of the calculations. We can exclude the first concern because Kalman filter is recursive algorithm that is based on simple math operations (summation and multiplication). However, we investigate carefully not only the errors of estimated SOC values but time of their evaluation as well. The obtained results are presented in the figure below.
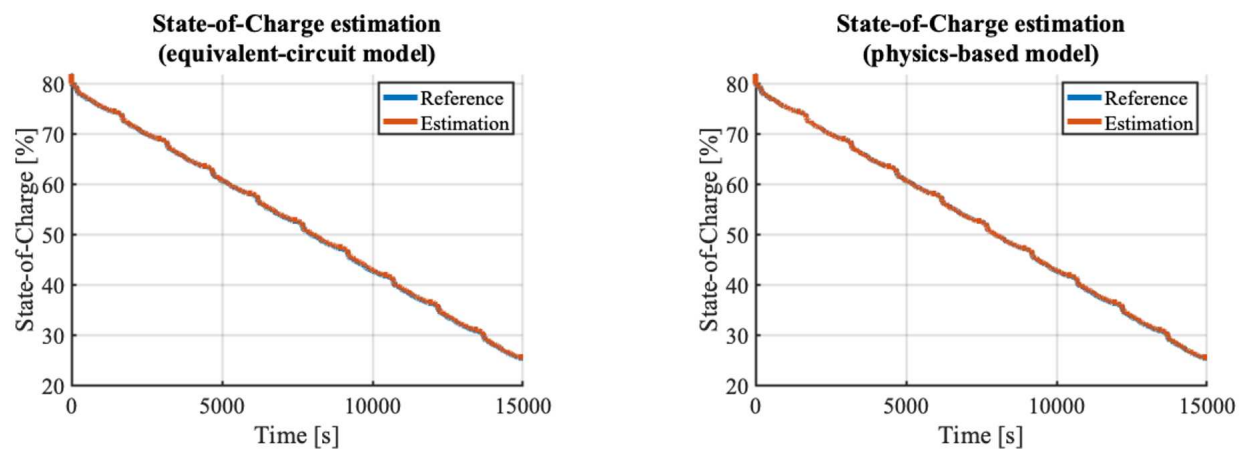

F i g. $\quad 5.2$

Results of SOC estimation by means of Kalman filter

It is indistinguishable from the figure above what algorithm provided the best result. Results are practically the same and RMS SOC-estimation error equals to $0.4 \%$ for ECM based estimation and $0.36 \%$ for physics-based one (see figure below).
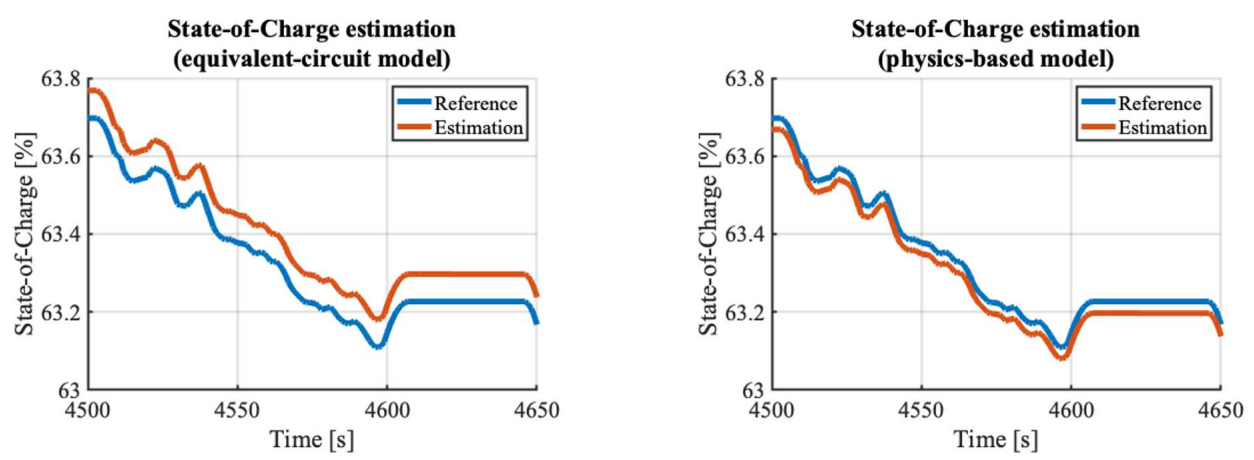

F i g. $\quad 5.3$

Results of SOC estimation by means of Kalman filter

Furthermore, results are comparable at the time of calculation. It takes $5.4 \mathrm{~s}$ to calculate 15000 values of SOC by means of ECM based Kalman filter and $5.1 \mathrm{~s}$ by physics-based approach for the same number of data samples.

However, physics-based approach is more powerful tool with regards to other lithium-ion cell states. While ECM-based Kalman filter allows estimating of battery internal resistance 
and capacity, physics-based one provides estimation of electrochemical variables. Example of estimation of two electrochemical variables are presented in the figure below.

Despite the fact that values of internal resistance and capacity are enough to estimate lithium-ion battery $\mathrm{SOH}$, these values provide only quantitative estimation. Whereas knowledge of the estimated values of internal electrochemical variables gives us qualitative information of battery states.
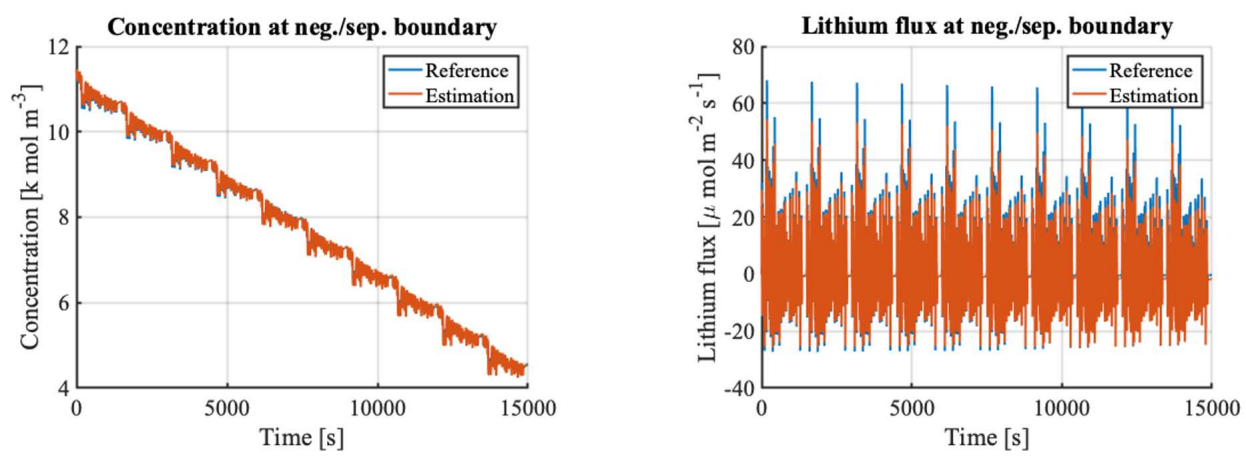

F i g. $\quad 5.4$

Results of internal variables estimation by means of physics-based model and Kalman filter

\section{Summary}

In the present paper we considered two different approaches to estimate states of lithium-ion cell: ECM-based and physics-based. We presented results of the estimation for one particular lithium-ion battery cell, and compared them in terms of RMS errors and time required for the calculations. Obtained results met our expectations: ECM-based and physics-based approaches demonstrated comparable accuracy and computational complexity. However, we made a conclusion that physics-based approach is more powerful tool with regard to estimation of the cell $\mathrm{SOH}$ because it allows estimating of the internal battery variables.

Obtained results are important for our further research that will lie in battery control topics. We would like to build optimal battery control to slow rate of battery ageing with regards to constraints on the electrochemical variables.

\section{Nomenclature}

$A$ : surface area of the porous electrode $\left[\mathrm{m}^{2}\right]$

$a_{s}$ : specific surface area of the porous electrode $\left[\mathrm{m}^{-1}\right]$

$\alpha$ : charge-transfer coefficient

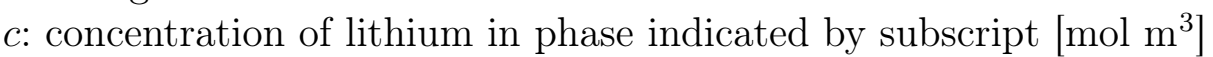

$c_{e, 0}$ : steady-state concentration of lithium in the electrolyte phase $\left[\mathrm{mol} \mathrm{m}^{3}\right]$

$c_{s, \text { max }}$ : maximum lithium concentration in an electrode particle $\left[\mathrm{mol} \mathrm{m}{ }^{3}\right]$

$c_{s, 0}$ : initial concentration of lithium in the solid phase $\left[\mathrm{mol} \mathrm{m}{ }^{3}\right]$

$c_{s, e}$ : surface concentration of lithium in a spherical electrode particle $\left[\mathrm{mol} \mathrm{m}{ }^{3}\right]$

$D_{\text {e,eff: }}$ effective electrolyte diffusivity $\left[\mathrm{m}^{2} \mathrm{~s}^{-1}\right]$

$D_{s}$ : solid diffusivity $\left[\mathrm{m}^{2} \mathrm{~s}^{-1}\right]$

$\varepsilon$ : volume fraction of phase indicated by subscript

$f_{ \pm}$: mean molar activity coefficient

$F$ : Faraday constant $\left[\mathrm{C} \mathrm{mol}^{-1}\right]$

$\phi$ : potential of the phase indicated by subscript [V]

$I$ : applied current density $\left[\mathrm{A} \mathrm{m}^{2}\right]$ 
$i_{\text {app }}:$ applied cell current $[\mathrm{A}]$

$j$ : reaction flux $\left[\mathrm{mol} \mathrm{m} \mathrm{m}^{2} \mathrm{~s}^{-1}\right]$

$k$ : rate constant for the electrochemical reaction $\left[\mathrm{mol}^{\alpha-1} \mathrm{~m}^{4-3 \alpha} \mathrm{s}^{-1}\right]$

$\kappa_{\text {eff }}$ : effective electrolyte conductivity $\left[\mathrm{S} \mathrm{m}^{-1}\right]$

$L$ : length of the cell $[\mathrm{m}]$

$\eta$ : local overpotential [V]

$r$ : radial coordinate $[\mathrm{m}]$

$R$ : universal gas constant $\left[\mathrm{J}^{-1} \mathrm{~mol}^{-1} \mathrm{~K}^{-1}\right]$

$R_{\mathrm{film}}$ : film resistance $\left[\Omega \mathrm{m}^{2}\right]$

$R_{s}$ : particle radius $[\mathrm{m}]$

$\sigma_{\text {eff: }}$ effective solid conductivity $\left[\mathrm{S} \mathrm{m}^{-1}\right]$

$T$ : temperature $[\mathrm{K}]$

$t_{+}^{0}$ : transference number

$U_{\text {ocp }}$ : open circuit potential [V]

$x$ : 1D linear coordinate across the cell $[\mathrm{m}]$

\section{Subscripts}

$e$ : pertaining to the electrolyte phase

$s$ : pertaining to the solid phase

\section{REFERENCES}

1. V. Pop et al., Battery management systems: Accurate state-of-charge indication for battery-powered applications, Springer Science \& Business Media, 2008.

2. M. Murnane et al., "A closer look at state of charge (SOC) and state of health (SOH) estimation techniques for batteries", 2017.

3. S. Nejad et al., "A systematic review of lumped-parameter equivalent circuit models for real-time estimation of lithium-ion battery states", Journal of Power Sources, 316 (2016), $183-196$.

4. A. Farmann et al., "A comprehensive review of on-board State-of-Available-Power prediction techniques for lithium-ion batteries in electric vehicles", Journal of Power Sources, 329 (2016), 123-137.

5. S. Nejad et al., "Enhanced state-of-charge estimation for lithium-ion iron phosphate cells with flat open-circuit voltage curves", IECON 2015-41st Annual Conference of the IEEE Industrial Electronics Society, 2015, 003187-003192.

6. T. Xidong et al., "Capacity estimation for Li-ion batteries", Proceedings of the 2011 American Control Conference, 2011, 947-952.

7. R.R. Richardson et al., "Gaussian process regression for forecasting battery state of health", Journal of Power Sources, 357 (2017), 209-219.

8. X. Zhang et al., "A novel method for lithium-ion battery state of energy and state of power estimation based on multi-time-scale filter", Applied energy, 216 (2018), 442-451.

9. K. A. Smith et al., "Control oriented 1D electrochemical model of lithium ion battery", Energy Conversion and management, 48:9 (2007), 2565-2578. 
10. T. F. Fuller et al., "Simulation and optimization of the dual lithium ion insertion cell", Journal of the Electrochemical Society, 141:1 (1994), 1-10.

11. G. L. Plett, "Recursive approximate weighted total least squares estimation of battery cell total capacity", Journal of Power Sources, 196:4 (2011), 2319-2331.

12. R. Ahmed et al., "Model-based parameter identification of healthy and aged li-ion batteries for electric vehicle applications", SAE International Journal of Alternative Powertrains, 4:2 (2015), 233-247.

13. F. Zhang et al., "State-of-charge estimation based on microcontroller-implemented sigmapoint kalman filter in a modular cell balancing system for lithium-ion battery packs", 2015 IEEE 16th Workshop on Control and Modeling for Power Electronics (COMPEL), 2015, $1-7$.

14. S. Zhao et al., "Global sensitivity analysis of battery equivalent circuit model parameters", 2016 IEEE Vehicle Power and Propulsion Conference (VPPC), 2016, 1-4.

15. G. L. Plett, Battery management systems. V.II: Equivalent-circuit methods, Artech House, 2015.

16. M. Doyle et al., "Comparison of modeling predictions with experimental data from plastic lithium ion cells", Journal of the Electrochemical Society, 143:6 (1996), 1890-1903.

17. J. L. Lee et al., "One-dimensional physics-based reduced-order model of lithium-ion dynamics", Journal of Power Sources, 220 (2012), 430-448.

18. Di D. Domenico et al., "Lithium-ion battery state of charge and critical surface charge estimation using an electrochemical model-based extended Kalman filter", Journal of dynamic systems, measurement, and control, 132:6 (2010), 061302.

19. K.D. Stetzel et al., "Electrochemical state and internal variables estimation using a reduced-order physics-based model of a lithium-ion cell and an extended Kalman filter", Journal of Power Sources, 278 (2015), 490-505.

20. J. L. Lee et al., "Discrete-time realization of transcendental impedance models, with application to modeling spherical solid diffusion", Journal of Power Sources, 206 (2012), $367-377$.

Submitted 7.03.2019 